\title{
Correction to: Development of a new reproductive tissue cryopreservation clinical service for children: the Oxford programme
}

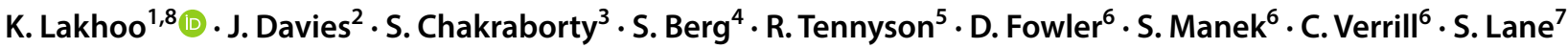

Published online: 6 February 2020

c) Springer-Verlag GmbH Germany, part of Springer Nature 2020

\section{Correction to: \\ Pediatric Surgery International (2019) 35:1271-1278 \\ https://doi.org/10.1007/s00383-019-04503-3}

In the published version, the Acknowledgements section was missing a funding note of co-author Dr C Verrill. The corrected version should read as follows.

\section{Acknowledgements}

Prof. C Becker as our liaison for young adult transition care. The research was supported by the National Institute for Health Research (NIHR) Oxford Biomedical Research Centre (BRC). The views expressed are those of the author(s) and not necessarily those of the NHS, the NIHR or the Department of Health.

The original article can be found online at https://doi.org/10.1007/ s00383-019-04503-3.

K. Lakhoo

Kokila.lakhoo@nds.ox.ac.uk

1 Department of Paediatric Surgery, University of Oxford and Oxford University Hospitals, Oxford, UK

2 Oxford Tissue Bank, University of Oxford and Oxford University Hospitals, Oxford, UK

3 Department of Paediatric Radiology, University of Oxford and Oxford University Hospitals, Oxford, UK

4 Department of Paediatric Anaesthesia, University of Oxford and Oxford University Hospitals, Oxford, UK

5 Department of Psychology, University of Oxford and Oxford University Hospitals, Oxford, UK

6 Department of Cellular Pathology, University of Oxford and Oxford University Hospitals, Oxford, UK

7 Department of Paediatrics and Child Health, University of Oxford and Oxford University Hospitals, Oxford, UK

8 Nuffield Department of Surgery, Oxford University and Oxford University Hospitals, Headley Way,

Oxford OX39DA, UK 